\title{
Infection dynamics of the monogenean parasite Gyrodactylus gasterostei on sympatric and allopatric populations of the three-spined stickleback Gasterosteus aculeatus
}

\author{
Joost A.M. Raeymaekers ${ }^{1}$, K. Mathias Wegner ${ }^{2,3}$, Tine Huyse ${ }^{1}$ and Filip A.M. Volckaert ${ }^{1}$ \\ ${ }^{1}$ Laboratory of Animal Diversity and Systematics, Katholieke Universiteit Leuven, Ch. Deberiotstraat, 32, B-3000 Leuven, \\ Belgium; \\ ${ }^{2}$ Department of Evolutionary Ecology, Max-Planck Institute for Evolutionary Biology, August-Thienemann-Strasse 2, D-24306 \\ Plön, Germany; \\ ${ }^{3}$ Present address: Leibniz Institute for Marine Sciences (IFM-GEOMAR), Evolutionary Ecology of Marine Fishes, Düsternbro- \\ ker Weg 20, D-24103 Kiel \& Alfred Wegener Institute for Polar and Marine Research, Waddenseastation Sylt, Hafenstrasse 43, \\ D-25992 List/Sylt, Germany
}

\begin{abstract}
Parasites with high host specificity maximally depend on their hosts, which should increase the likelihood of coevolution. However, coevolution requires reciprocal selection exerted by the host and the parasite, and thus a considerable level of parasite virulence. In species of the monogenean ectoparasite genus Gyrodactylus consecutive generations are confronted with a single host, which may constrain the evolution of virulence. Transmission, which is often important in the ecology of Gyrodactylus species, may have the opposite effect, but may also lead to the avoidance of coevolutionary arms races. We investigated the potential outcome of coevolution between Gyrodactylus gasterostei Gläser, 1974 and its host, the three-spined stickleback (Gasterosteus aculeatus L.) by determining the strength of genotype by genotype $(\mathrm{G} \times \mathrm{G})$ interactions on two levels: within and between sympatric and allopatric host populations. To do so, we compared the parasite's infection dynamics on laboratory-reared sympatric (Belgian) and allopatric (German) hosts. We found that a parasite line successfully infected a range of sympatric host genotypes (represented by families), while it failed to establish on allopatric hosts. Phylogeographic studies suggest that neutral genetic divergence between the host populations cannot explain this dramatic difference. Provided that this result can be generalised towards other parasite lines, we conclude that coevolution in this host-parasite system is more likely to lead to local adaptation on the population level than to $\mathrm{G} \times \mathrm{G}$ interactions within populations.
\end{abstract}

Keywords: Monogenea, Gyrodactylus gasterostei, ecological immunity, fish parasite, Gasterosteus aculeatus, host specificity, local adaptation, parasite-host coevolution

Resistance against parasites is shaped by the antagonistic interaction among hosts and parasites. This interaction may result in coevolution between host and parasite, producing patterns like local adaptation, genotype by genotype $(\mathrm{G} \times \mathrm{G})$ interactions, and reciprocal selection (Woolhouse et al. 2002, Webster et al. 2004). All of these facets of antagonistic coevolution have been described in a number of host-parasite systems. For example, negative frequency-dependent selection causing Red Queen dynamics were driving local and temporal adaptation in hostparasite systems with direct transmission such as Daphnia magna and its microparasites (Ebert 1994, Decaestecker et al. 2007). Local adaptation was also observed in parasites with more complex life cycles (Ballabeni and Ward 1993, Lively and Dybdahl 2000). Similarly, reciprocal selection has been demonstrated in feather lice completing their life cycle directly on pigeon hosts (Clayton et al.
1999), but also in parasites with intermediate hosts such as Schistosoma species (Webster et al. 2004).

These examples demonstrate that coevolution is possible, irrespective of the parasite's life cycle. The role of host specificity for local adaptation, on the other hand, has rarely been investigated. In this study, we were interested in coevolution of parasites characterised by high host specificity and a direct life cycle. Such parasites are confronted with few selective forces other than the ones imposed by their hosts, and this close interaction should maximise the likelihood of coevolution. Coevolution is most likely observed when a specialist pathogen exerts a strong selection pressure on its host and vice versa (Woolhouse et al. 2002). This can lead to strong $\mathrm{G} \times \mathrm{G}$ interactions and maintain host and parasite genotypic diversity by negative frequency-dependent selection. However, when consecutive generations of parasites are confronted 
with genetically similar hosts (as is often the case in hostspecific parasites with a direct life cycle), coevolution may arise without the need for high virulence (Agrawal 2006). Relaxation of parasite selection for host counteradaptations might then lead to generalist strategies in parasites leading to comparable infection success on larger groups of related genotypes up to the whole population.

A group of parasites combining high host specificity with a direct life cycle is the flatworm genus Gyrodactylus (Monogenea, Platyhelminthes). Gyrodactylids represent common ectoparasites of fish species, living mainly on fins and gills. Embryos of Gyrodactylus species develop within each other's uterus like Russian dolls, allowing relatively short generation times and exponential population growth (Cable and Harris 2002). Such features parallel those of directly transmitted microparasites, for which substantial theories on the likelihood and consequences of coevolution have been developed and tested (Woolhouse et al. 2002). In contrast, data on the interaction of Gyrodactylus species and their hosts at the micro-evolutionary level are scarce, and the consequences for coevolution are unknown. Boeger et al. (2005) suggested that transmission has the potential to minimize coevolutionary arms races in Gyrodactylus species and their hosts. Transmission, for instance by direct host contact, indeed plays a central role in the biology of Gyrodactylus species. Other studies have focussed on the susceptibility of different species and populations of fishes, as infection with Gyrodactylus species may have severe pathological consequences (Soleng and Bakke 1998, Bakke et al. 1999, 2002). Van Oosterhout et al. (2003) found marked variation in resistance for Gyrodactylus species between guppy populations facing high and low predation risk. Intra-specific variation in Gyrodactylus species is largely neglected in experiments. Given the economical damage of Gyrodactylus species in aquaculture (Nielsen and Buchmann 2001), empirical data on the evolution of virulence and coevolution are highly desirable.

In this study we explore the coevolutionary dynamics between Gyrodactylus gasterostei Gläser, 1974 and its principal host, the three-spined stickleback Gasterosteus aculeatus L. The Gyrodactylus community of sticklebacks in Europe includes several species, among which G. gasterostei and $G$. arcuatus are most commonly observed (Harris 1985, 1998). The epidemiology of G. gasterostei in Western Europe suggests narrow host specificity for the three-spined stickleback (Harris 1985, Raeymaekers et al. 2008a). Therefore, G. gasterostei can be considered a specialist and thus represents a suitable candidate to investigate the impact of host specialisation on the potential outcome of coevolution. Our experiment was carried out to compare the fitness (i.e., infection success and population growth) of an isogenic (i.e., derived from a single worm) G. gasterostei line on three laboratory-reared (i.e., immunologically naive) sibships from its sympatric Bel- gian host population, and three such sibships from an allopatric host population from northern Germany. Since consecutive generations of worms are usually confronted with the same or closely related hosts, it is possible that $\mathrm{G} \times \mathrm{G}$ interactions are strong. Alternatively, if virulence is low, $\mathrm{G} \times \mathrm{G}$ interactions may be weak and coevolution may be weak as well. Consequently, we wanted to test whether parasites may have adopted a generalist strategy and have comparable infection success throughout the whole host population or even the entire host species. Furthermore, genetic variation in resistance of the sympatric and the allopatric host population was compared to neutral genetic divergence using a $\mathrm{Q}_{\mathrm{ST}}-\mathrm{F}_{\mathrm{ST}}$ approach (Merilä and Crnokrak 2001). This was done to assess whether or not the differences in infectivity and infection intensity were most likely caused by selection on resistance of the hosts or local adaptation of the parasite, rather than by neutral divergence of the host populations.

\section{MATERIALS AND METHODS}

Source material. The experimental infections were performed with a Gyrodactylus gasterostei line collected from a single three-spined stickleback stemming from Westkerke (Belgium; $3^{\circ} 00^{\prime} \mathrm{E}, 51^{\circ} 10^{\prime} \mathrm{N}$ ). This site represents a small eutrophic polder creek near the coast, with a very slow freshwater current and a high density of macrophytes. The stickleback population here, i.e., the sympatric host population, belongs to the lowland ecotype (Raeymaekers et al. 2005, 2007, Van Dongen et al. 2009; Fig. 1). Gyrodactylus gasterostei is the most common Gyrodactylus species on this host population (Raeymaekers et al. 2008a). A German stickleback population from Vierer See (Plön, Germany; $10^{\circ} 25^{\prime} \mathrm{E}, 54^{\circ} 09^{\prime} \mathrm{N}$; Fig. 1), a lake draining into the Baltic Sea, was selected as the allopatric host population because of the common occurrence of G. gasterostei (Kalbe M., M.P.I.-Plön, Germany; pers. comm.).

The experiment was performed with the $\mathrm{F}_{1}$ offspring derived from crosses from sympatric Belgian (B) or allopatric German (G) fish, caught in March 2003. These fish were disinfected with a 1:8000 formalin solution and crossed in the laboratory in July 2003. This resulted in three sympatric $F_{1}$ sibships, coded B1, $\mathrm{B} 2$ and $\mathrm{B} 3$, and three allopatric $\mathrm{F}_{1}$ sibships, coded G1, G2 and G3. After spawning, clutches were collected and kept in aerated glass jars until hatching, before fry were transferred to 20-1 flowthrough aquaria. These fish reached maturity in spring 2004 and were then used for our experiment (see below). Prior to the experiment, they were never exposed to the parasite and can therefore be regarded as immunologically naive.

Experimental design. The experimental design is shown in Fig. 2. All fish were kept individually (see below). The infection experiment was preceded by a worm breeding stage, in order to obtain sufficiently large numbers of worms. In spring 2004, the Westkerke site was revisited to catch infected three-spined sticklebacks. These donor fish were transported alive to the laboratory, and their worms were transmitted within 1-2 days to naive fish of family B1, i.e., one of the sympatric families. The required number of worms was reached by passaging one isogenic line (i.e., starting from a single worm) on four subsequent sets of randomly selected B1 hosts. Set sizes during the 
four rounds were $\mathrm{n}=1,10,32$ and 39 sticklebacks, with infections lasting 20, 30, 24 and 30 days, respectively (Fig. 2). The isogenic line was initially selected out of 10 lines as the one with the best growth on the first host and was later confirmed to be $G$. gasterostei, based on the ITS rDNA region encompassing the Internal Transcribed Spacers (ITS1 and ITS2) and the small ribosomal subunit (5.8S rRNA) gene (Zietara et al. 2002).

After the last passage, an experiment was initiated to compare the success of the parasite line on sibship B1 with its success on the other sympatric sibships (B2 and B3), and on the allopatric $F_{1}$ sibships (G1, G2 and G3). On day zero of the experiment, 16 fish of each sibship were infected. The infections were organised such that the worms from each final worm breeding host were distributed equally over the six sibships (B1-B3, G1-G3). The experiment was evaluated by anaesthetising all fish weekly to count the number of worms.

Infection and fish maintenance. Fish used for worm breeding and experimental fish were briefly anaesthetized with $50 \mathrm{mg} \mathrm{l}^{-1} \mathrm{MS} 222$ and infected by putting a G. gasterostei individual on the right pectoral fin, following standard methods of Cable et al. (2000). Subsequently, all fish were placed individually in 2-1 aquaria, which were positioned randomly in a controlled cooling system at $12^{\circ} \mathrm{C}$ and a $12 \mathrm{~h}$ light:dark photoperiod. Fish were fed three times a week a mix of brine shrimp Artemia salina nauplii and bloodworms (chironomid larvae). Water was changed weekly using dechlorinated tap water.

Feeding activity. Feeding activity was registered at the beginning of the infection experiment and 5 weeks later as an indicator of the health of the sticklebacks. It was measured as the number of feeding lunges made at Artemia salina nauplii during $1 \mathrm{~min}$ averaged over two trials on consecutive days. This test was performed on all experimental fish by placing them in their individual aquarium in a light-tight observation box, illuminated from above. After $5 \mathrm{~min}$, a standardised number of nauplii $\left(>1000\right.$ ind. $\left.1^{-1}\right)$ were introduced and feeding lunges were counted by a single observer (J.A.M. Raeymaekers). Feeding activity is considered to be a good health indicator in guppies (Van Oosterhout et al. 2003). We found this measure to be correlated between the two consecutive days (Pearson correlation $=0.51$; $\mathrm{P}<0.0001$ ). At the beginning of the experiment, there were no significant differences in feeding activity among populations (fixed effect; $\mathrm{F}_{1,90}=3.29, \mathrm{P}=0.20$ ) or among sibships nested in population (random effect; $\mathrm{F}_{4.90}=1.41, \mathrm{P}=0.24$ ). The average feeding activity here was $10.71 \pm 6.64$ lunges per minute.

Data analysis. Stickleback feeding activity after 5 weeks was evaluated as above, i.e., we investigated with a general linear model whether the infection experiment had induced differential feeding activity among populations (considered as fixed effect) or among sibships nested in population (considered as random effect).

Total worm load was calculated as the $\log _{\mathrm{e}}$-transformed sum of weekly infection intensities. This sum always included the initial worm, making the logarithmic transformation always defined. A general linear model was performed on total worm load to investigate the effect of population (considered as fixed) and the effect of sibship nested in population (considered as random). To assess epidemiological differences over time, weekly infection intensity was modelled with a generalised linear mixed model (GLMM; Molenberghs and Verbeke 2005), including time as a fixed factor. Infection intensity was assumed to be in-

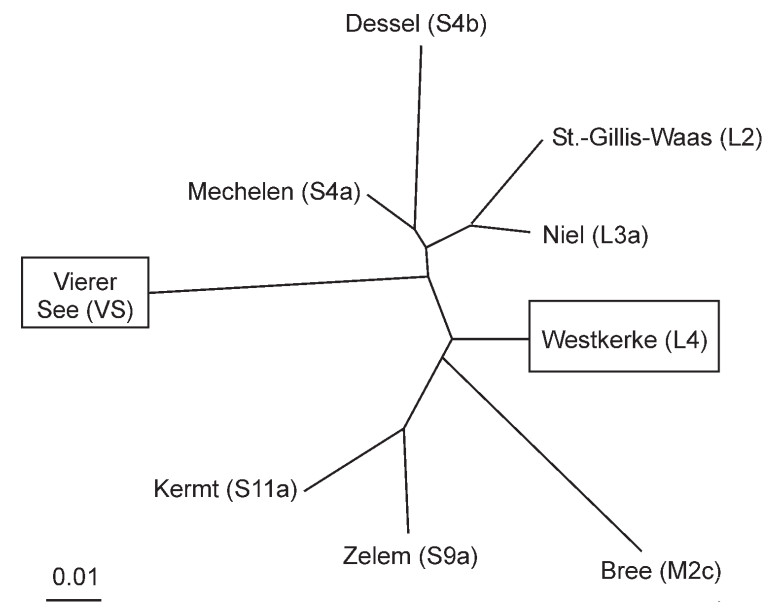

Fig. 1. Unrooted neighbour joining tree of Cavalli-Sforza and Edwards (1967) genetic distances (based on six microsatellite loci) among two experimental and seven neighbouring threespined stickleback Gasterosteus aculeatus populations, recalculated from Raeymaekers et al. (2007, 2008b) and Reusch et al. (2001). Experimental populations are Westkerke (observed heterozygosity $\left.\mathrm{H}_{\mathrm{O}}=0.81\right)$ and Vierer See $\left(\mathrm{H}_{\mathrm{O}}=0.64\right)$. Belgian populations belong to the upper Scheldt drainage (S), the upper Meuse drainage (M), or the coastal lowlands (L). Vierer See belongs to the prevailing lake clade found in northern Germany (Reusch et al. 2001).

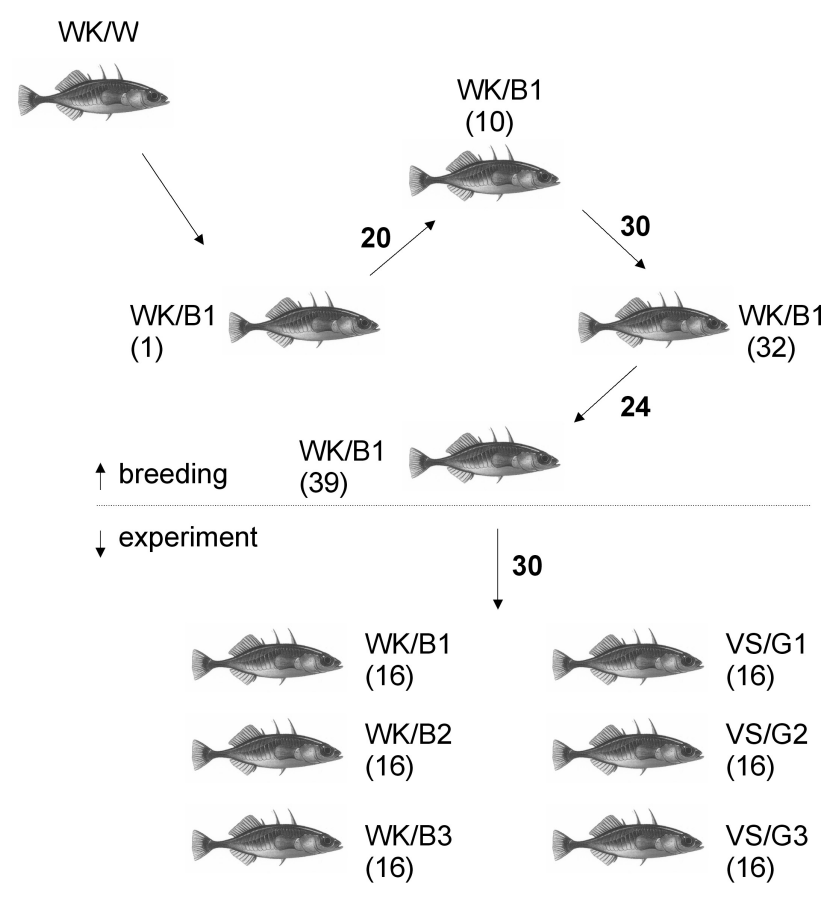

Fig. 2. Graphical presentation of worm breeding and experimental design. Codes indicate Gasterosteus aculeatus population/ genotype combinations. Populations are Westkerke (WK) and Vierer See (VS), and genotype codes refer to sibship B1, B2, B3, G1, G2 and G3, or wild individuals (W). Numbers in bold along arrows represent days of infection before passaging. Numbers in parentheses represent sample size of fish, which were kept individually in 2-1 aquaria. 
dependently Poisson-distributed, conditionally on the random effects of the repeatedly measured individual hosts. Except for the GLMMs, which were executed in PROC GLIMMIX in SAS 9.1, all analyses were performed in STATISTICA 6.0.

Differences in infection parameters and feeding activity between the sympatric and the allopatric host population were compared with neutral genetic divergence using a $\mathrm{Q}_{\mathrm{ST}}-\mathrm{F}_{\mathrm{ST}}$ approach. This was done to assess whether genetic variation in resistance and feeding activity was larger or smaller than expected based on the genetic differentiation among the host populations. $\mathrm{F}_{\mathrm{ST}}$, estimated as $\theta$ (Weir and Cockerham 1984), quantifies the proportion of among-population genetic variance in neutral markers, and was recalculated from Raeymaekers et al. (2007) and Reusch et al. (2001) based on six microsatellite loci with the software GENETIX 4.04 (Belkhir et al. 2002). This dataset included 47 individuals from the Belgian host population, and 27 individuals from the German host population. A 95\% confidence interval for $\mathrm{F}_{\mathrm{ST}}$ was obtained with a bootstrap over loci. $\mathrm{Q}_{\mathrm{ST}}$ (Spitze 1993) quantifies the proportion of among-population genetic variance in quantitative traits, and was calculated using a Bayesian estimation procedure implemented in the MCMCglmm library in R (Hadfield 2010). We used an animal model to calculate the within population additive genetic variance components of a Poisson model with total worm load as response or a Gaussian model with feeding activity as response. Confidence envelopes were estimated as $95 \%$ confidence intervals of 1000 random samples from the posterior distribution (MCMC chain length 250000, burnin removed 50000, thinning interval 200).

\section{RESULTS}

The experimental infections did not induce host mortality. After 5 weeks, fish from different sibships within populations displayed differential feeding activity (sibships nested in population: $\left.\mathrm{F}_{4,88}=3.61, \mathrm{P}=0.0090\right)$. In particular, sibship B1 showed a lower activity (5.53 \pm 4.39 lunges min. $\left.^{-1}\right)$ than sibship B2 (10.94 \pm 7.26 lunges min. $\left.{ }^{-1}\right)$ and sibship B3 (11.78 \pm 6.69 lunges min. $\left.^{-1}\right)$. As will be seen below, these differences cannot be attributed to the experimental infection. Furthermore, fish from different populations did not differ in feeding activity $\left(\mathrm{F}_{1,88}=1.53\right.$, $\mathrm{P}=0.28)$.

Overall, the number of infected individuals gradually decreased from $77 \%$ after the first week towards $20 \%$ after 6 weeks. From the start of the experiment allopatric sibships showed a remarkably low susceptibility compared to sympatric sibships $(31 \%$ and $6 \%$ initial resistance, respectively). Infection intensity peaked after 5 weeks for sympatric sibships (average worm load \pm S.E.: $17.81 \pm 3.45$ worms; max. 90) (Fig. 3), whereas maximal infection was already reached on allopatric sibships after the first week (average worm load \pm S.E.: $1.46 \pm 0.21$ worms; max. 5).

Total worm load was significantly lower on the allopatric host population than on the sympatric host population, but did not differ between sibships within populations (Table 1; Fig. 4). A repeated Poisson regression analysis on weekly infection intensities generated a significant
Table 1. ANOVA table of log-transformed total worm load on a sympatric and an allopatric three-spined stickleback Gasterosteus aculeatus population after 6 weeks of infection with an isogenic Gyrodactylus gasterostei line. Host sibship (nested in population) was included as a random effect. Significant P-values are in bold.

\begin{tabular}{lrrrrrrr}
\hline Effect & \multicolumn{1}{c}{ SS } & \multicolumn{7}{c}{ dfeffect } & MSeffect & dferror & MSerror & \multicolumn{1}{c}{ F } & P \\
\hline Intercept & 417.01 & 1 & 417.01 & 4 & 2.62 & 159.10 & $\mathbf{0 . 0 0 0 2}$ \\
$\begin{array}{l}\text { Sibship } \\
\text { (population) }\end{array}$ & 10.49 & 4 & 2.62 & 88 & 1.75 & 1.50 & 0.2099 \\
Population & 89.42 & 1 & 89.42 & 4 & 2.62 & 34.12 & $\mathbf{0 . 0 0 4 3}$ \\
Error & 154.06 & 88 & 1.75 & & & & \\
\hline
\end{tabular}

sibship by time interaction, pointing to a faster decline on sibship B1 at the end of the experiment $\left(\mathrm{F}_{10,223}=5.92\right.$; $\mathrm{P}<0.0001$, Fig. 3). Differences in total worm load between the sympatric and the allopatric host population, quantified as $\mathrm{Q}_{\mathrm{ST}}$, dramatically exceeded neutral genetic divergence $\left(\mathrm{Q}_{\mathrm{ST}}=0.96,95 \% \quad \mathrm{CI}=[0.68-0.99]\right.$; $\mathrm{F}_{\mathrm{ST}}=0.14,95 \% \mathrm{CI}=[0.08-0.21]$, Fig. 5). This indicates that selection on resistance of German hosts or strong local adaptation of the parasite in Belgium or a combination of both shaped this trait, rather than neutral divergence of the host populations. The observed differences in feeding activity, on the other hand, were rather caused by neutral divergence than selection, because the most probable value of genetic differentiation of this trait did not exceed neutral expectations (Fig. 5).

\section{DISCUSSION}

This study documents the results of an infection experiment comparing infectivity and infection intensity of the monogenean ectoparasite Gyrodactylus gasterostei between its sympatric and an allopatric host population. The isogenic parasite line used here tended to show weak genotype by genotype $(\mathrm{G} \times \mathrm{G})$ interactions on sympatric immunogenetically naive hosts, with hosts from the family used for worm rearing (B1) displaying lower infection intensities over time (Fig. 3). The main variation, however, was observed between the sympatric and the allopatric host population (Figs. 3, 4). The difference in total worm load between both populations was most likely caused by selection, as genetic differentiation for this trait $\left(\mathrm{Q}_{\mathrm{ST}}\right)$ by far exceeded neutral genetic divergence (Fig. 5). Differences in feeding activity, on the other hand, showed no stronger differentiation than expected under neutral divergence, indicating that little adaptive variation is present for that trait. Wide confidence envelopes of $\mathrm{Q}_{\mathrm{ST}}$ estimates are not surprising, as only a very small number of families was used to assess genetic variation. The point estimate of highest probability for the difference in susceptibility nevertheless reflects a clear pattern of selection when reflected against the close clustering of stickleback populations from Belgium and northern Germany (including Vierer See) based on microsatellite (Fig. 1) and 
Belgian families

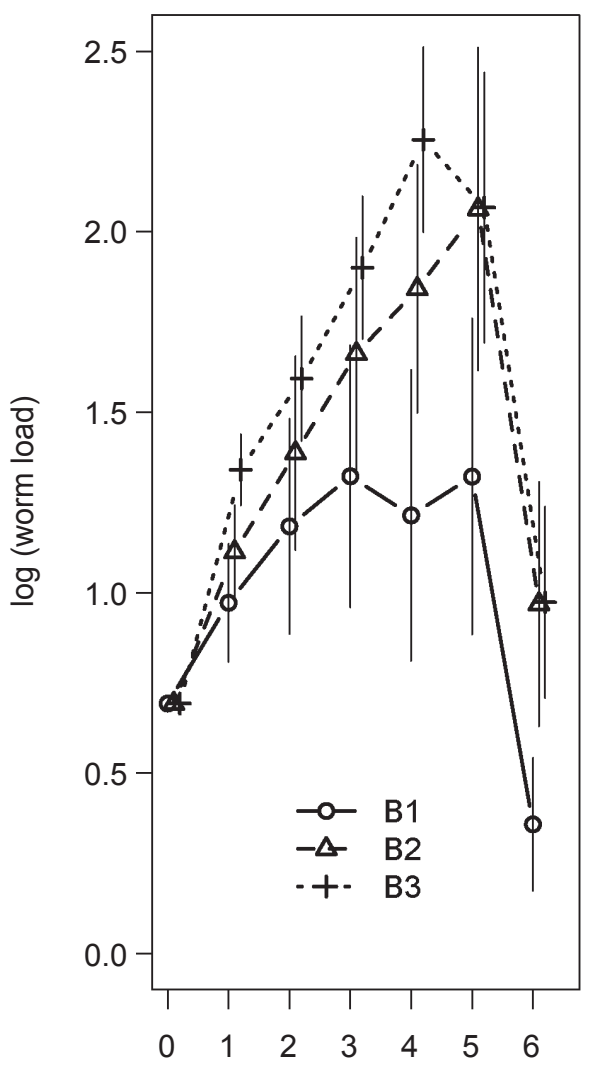

German families

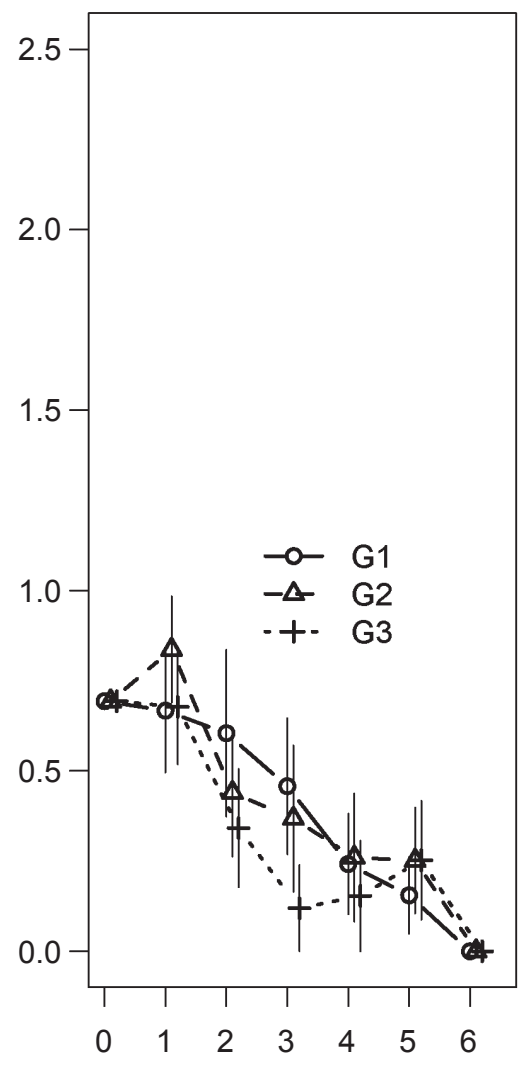

Weeks after infection

Fig. 3. Temporal dynamics of Gyrodactylus gasterostei infection intensities on three sympatric Belgian (B1, B2, B3 - left panel) and three allopatric German (G1, G2, G3 - right panel) sibships of Gasterosteus aculeatus. Worms were initially reared on fish from sibship B1 (solid line, left panel). Error bars indicate standard errors.

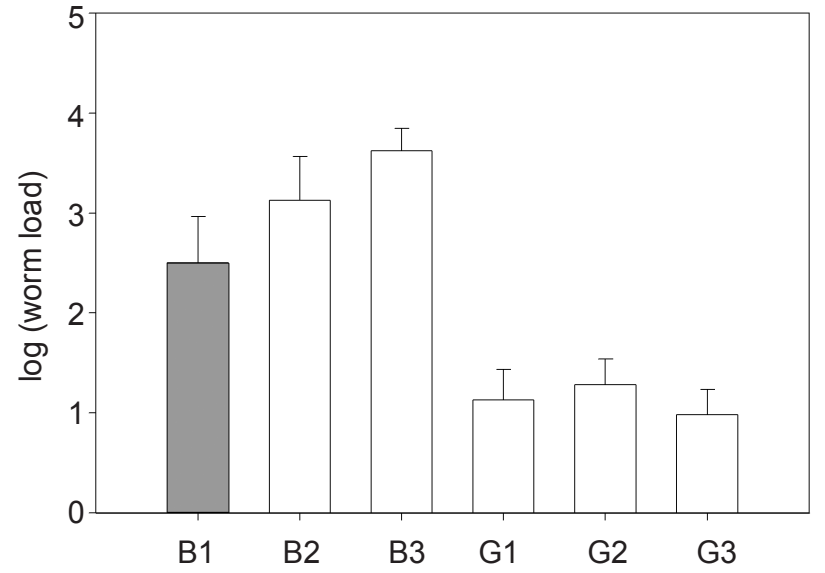

Fig. 4. Average log-transformed total Gyrodactylus gasterostei load in three sympatric Belgian (B1, B2, B3) and three allopatric German (G1, G2, G3) sibships of Gasterosteus aculeatus. Worms were initially reared on fish from sibship B1 (shaded bar). Error bars indicate standard errors.

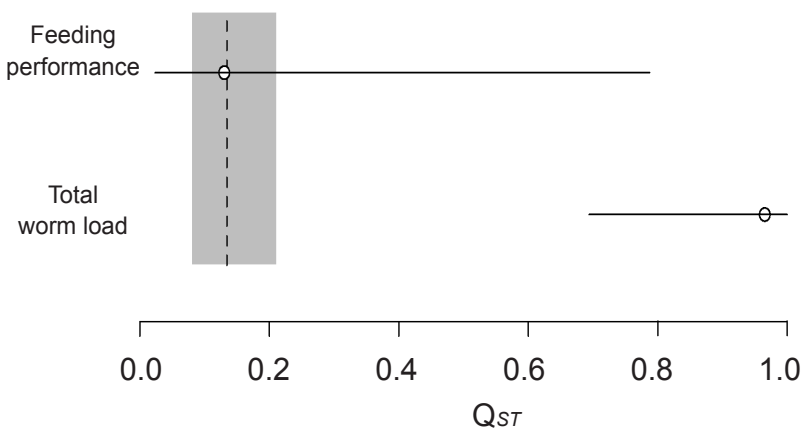

Fig. 5. Population differentiation between sympatric Belgian and allopatric German populations measured as $\mathrm{Q}_{\mathrm{ST}}$ for susceptibility (total worm load) and life history (feeding activity) traits. Points show the highest posterior density of 1000 random samples from the posterior distribution and lines show $95 \%$ confidence intervals of the posterior distribution. Phenotypic differentiation is compared to neutral genetic divergence measured as $\mathrm{F}_{\mathrm{ST}}$ (dashed line) and its $95 \%$ C.I. (shaded area). 
mitochondrial (Mäkinen et al. 2006, Mäkinen and Merila 2008) phylogeographic studies. In summary, our results suggest that individual parasite lines are rather adapted to local host populations than to specific host genotypes.

Generalisation of these conclusions is difficult mainly for two reasons. Firstly, although this is the first study testing for differences in susceptibility for a Gyrodactylus species between sympatric and allopatric host populations, we only tested a single parasite line. It is possible that parasite lines are highly variable with respect to their specificity for host genotypes and host populations. Secondly, the experiment did not allow for a formal test of local adaptation, as we did not include the reciprocal sympatric and allopatric combinations (Kaltz and Shykoff 1998, Kawecki and Ebert 2004). Therefore, the strong genetic differentiation in resistance can be explained by higher immune competence of German hosts (Scharsack et al. 2007), local adaptation of the worm isolate to Belgian hosts, or a combination of both. It is remarkable here that the allopatric host appeared highly unsuitable, as most worms died in the first week, after giving birth only once. This observation requires further investigation.

Despite of these shortcomings, the experiment nevertheless suggested that the virulence of $G$. gasterostei is comparatively low. Worms induced no host mortality, and host feeding activity was not related to worm load. Low pathogenicity seems to be characteristic of G. gasterostei, even when infecting naive three-spined stickleback populations (de Roij et al. 2011). However, virulence may also be highly dependent on environmental conditions (Wegner et al. 2008), and will not necessarily be expressed under benevolent laboratory conditions. In the case of $G y$ rodactylus species, population growth rate is particularly sensitive to environmental factors (Bakke et al. 2002), the hormonal status of the host (Harris et al. 2000) and food availability (Kolluru et al. 2006). In the case of G. gasterostei, it has been shown that populations of worms grow larger on weak or stressed hosts than on healthy fish (de Roij et al. 2011). While some congeners can be highly virulent (e.g. G. salaris; Bakke et al. 1999), virulence of G. gasterostei in good conditions seems not sufficient to drive the evolution of host defences.

Next to virulence, the presence of genetic variation in host susceptibility and parasite infectivity is another prerequisite for coevolution. In general, fishes have been shown to exhibit heritable variation or apparent differences in susceptibility to Gyrodactylus species (Madhavi and Anderson 1985, Bakke et al. 1999, Van Oosterhout et al. 2003). We now know that variation in resistance to G. gasterostei is present among host populations (de Roij et al. 2011; this study). Furthermore, a potential genetic basis for variation in resistance at the individual level has been identified by showing an association between the prevalence of $G$. gasterostei and a single class IIb allele of the major histocompatibility complex in a wild, riverine stickleback population (Eizaguirre et al. 2009). In our experiment, the breeding of the parasites was performed in a way that maximised the interaction with a single sympatric host sibship. Interestingly, the isolate tended to show lower infection intensities on this host sibship than on other sympatric host sibships. However, the differences were rather small, and since there were no directional changes in infection intensity during the four rounds of worm breeding (data not shown), these results suggest that $\mathrm{G} \times \mathrm{G}$ interactions within populations were rather weak when infected with this worm isolate. Such generalist strategy of the parasite facilitates parasite transmission, as unsuitable hosts will be rare. If these results also apply to other parasite isolates, then coevolution fuelled by $\mathrm{G} \times \mathrm{G}$ interactions might be of lesser importance in this host-parasite system.

\section{Conclusion}

In contrast to macroparasites with complex life cycles, consecutive generations of Gyrodactylus species are confronted with a single host genotype. Hence, Gyrodactylus species are expected to reach a balance between its need to reproduce and the cost of harming its host - similar to vertically transmitted parasites (Ebert and Herre 1996). We demonstrated that the fitness of laboratory-reared three-spined sticklebacks was largely unaffected following infection with an isogenic line of the monogenean parasite G. gasterostei. With such a low virulence, reciprocal selection pressures driving coevolutionary processes may be rather weak. Furthermore, the infections revealed small differences in susceptibility within host populations, but strong differences between host populations. Provided that this result can be generalised towards other parasite lines, we conclude that coevolution in this host-parasite system is more likely to lead to local adaptation on the population level than to $\mathrm{G} \times \mathrm{G}$ interactions within populations. Such a specificity level is in agreement with the central role of transmission in the ecology of Gyrodactylus species (Boeger et al. 2005). In general, Gyrodactylus species differ considerably in the level of host specificity, transmission capacity, and reproductive strategies (Harris 1993). These characteristics may influence the evolution of virulence, and may vary among closely related Gyrodactylus species on a single host species. Further exploration of this group of parasites, especially on small vertebrate hosts with reasonably short generation times like sticklebacks, guppies and gobies, may reveal how these characteristics affect the chances for coevolution to occur.

Acknowledgements. We thank S. Geldof, M. De Coninck, I. Hontis, E. Pape, B. Christiaen, J. Van Houdt and S. Usé for sampling and technical assistance. M. Kalbe, E. Decaestecker, J. de Roij and two anonymous referees provided helpful comments. G. Verbeke contributed with statistical advice. Research was sponsored by the Research Foundation - Flanders (postdoctoral fellowship to J.A.M.R. and T.H. and research project G.0142.03) and by the K.U.Leuven (project GOA/2006/06). 


\section{REFERENCES}

Agrawal A.F. 2006: Similarity selection and the evolution of sex: revisiting the Red Queen. PLoS Biol. 4: 1364-1371.

Bakke T.A., Harris P.D., Cable J. 2002: Host specificity dynamics: observations on gyrodactylid monogeneans. Int. J. Parasitol. 32: 281-308.

Bakke T.A., Soleng A., Harris P.D. 1999: The susceptibility of Atlantic salmon (Salmo salar L.) $\times$ brown trout (Salmo trutta L.) hybrids to Gyrodactylus salaris Malmberg and Gyrodactylus derjavini Mikailov. Parasitology 119: 467-481.

Ballabeni P., WaRd P.I. 1993: Local adaptation of the trematode Diplostomum phoxini to the European minnow Phoxinus phoxinus, its second intermediate host. Funct. Ecol. 7: 84-90.

Belkhir K., Borsa P., Chiкhi L., Raufaste N., Bonhomme F. 2002: Genetix 4.04: logiciel sous Windows TM pour la génétique des populations. Laboratoire Génome, Populations, Interactions, CNRS UMR 5000, Université de Montpellier II, Montpellier (France).

Boeger W.A., Kritsky D.C., Pie M.R., Engers K.B. 2005: Mode of transmission, host switching, and escape from the Red Queen by viviparous gyrodactylids (Monogenoidea). J. Parasitol. 91: 1000-1007.

Cable J., Harris P.D. 2002: Gyrodactylid developmental biology: historical review, current status and future trends. Int. J. Parasitol. 32: 255-280.

Cable J., Harris P.D., Bakke T.A. 2000: Population growth of Gyrodactylus salaris (Monogenea) on Norwegian and Baltic Atlantic salmon (Salmo salar) stocks. Parasitology 121: 621629.

Cavalli-Sforza L.L., Edwards A.W.F. 1967: Phylogenetic analysis: models and estimation procedures. Evolution 32: 550-570.

Clayton D.H., Lee P.L.M., Tompkins D.M., Brodie E.D. 1999: Reciprocal natural selection on host-parasite phenotypes. Am. Nat. 154: 261-270.

de Roij J., Harris P.D., MacColl A.D.C. 2011: Divergent resistance to monogenean flatworm among three-spined stickleback populations. Funct. Ecol. 25: 217-226.

Decaestecker E., Gaba S., Raeymaekers J.A.M., Stoks R., Van Kerckhoven L., Ebert D., De Meester L. 2007: Hostparasite 'Red Queen' dynamics archived in pond sediment. Nature 450: 870-873.

EBERT D. 1994: Virulence and local adaptation of a horizontally transmitted parasite. Science 265: 1084-1086.

Ebert D., Herre E.A. 1996: The evolution of parasitic diseases. Parasitol. Today 12: 96-101.

Eizaguirre C., Yeates S.E., Lenz T.L., Kalbe M., Milinski M. 2009: MHC-based mate choice combines good genes and maintenance of MHC polymorphism. Mol. Ecol. 18: 3316-3329.

HADFIELD J.D. 2010: MCMC methods for multi-response Generalized Linear Mixed Models: The MCMCglmm R package. J. Stat. Software 33: 1-22.

HARRIS P.D. 1985: Species of Gyrodactylus von Nordmann, 1832 (Monogenea: Gyrodactylidae) from freshwater fishes in southern England, with a description of Gyrodactylus rogatensis sp. nov. from the bullhead Cottus gobio L. J. Nat. Hist. 19: 791-809.

HARRIS P.D. 1993: Interactions between reproduction and population biology in Gyrodactylid monogeneans - a review. Bull. Fr. Peche Piscic. 328: 47-65.

HARRIS P.D. 1998: Ecological and genetic evidence for clonal reproduction in Gyrodactylus gasterostei Gläser, 1974. Int. J. Parasitol. 28: 1595-1607.
Harris P.D., Soleng A., Bakke T.A. 2000: Increased susceptibility of salmonids to the monogenean Gyrodactylus salaris following administration of hydrocortisone acetate. Parasitology 120: 57-64.

Kaltz O., Shy koff J.A. 1998: Local adaptation in host-parasite systems. Heredity 81: 361-370.

Kawecki T.J., Ebert D. 2004: Conceptual issues in local adaptation. Ecol. Lett. 7: 1225-1241.

Kolluru G.R., Grether G.F., South S.H., Dunlop E., Cardinali A., Liu L., Carapiet A. 2006: The effects of carotenoid and food availability on resistance to a naturally occurring parasite (Gyrodactylus turnbulli) in guppies (Poecilia reticulata). Biol. J. Linn. Soc. 89: 301.

Lively C.M., Dybdahl M.F. 2000: Parasite adaptation to locally common host genotypes. Nature 405: 679-681.

Madhavi R., ANderson R.M. 1985: Variability in the susceptibility of the fish host, Poecilia reticulata, to infection with Gyrodactylus bullatarudis (Monogenea). Parasitology 91: 531-544.

MäKinen H.S., Cano J.M., Merilä J. 2006: Genetic relationships among marine and freshwater populations of the European three-spined stickleback (Gasterosteus aculeatus) revealed by microsatellites. Mol. Ecol. 15: 1519-1534.

MäKinen H.S., Merilä J. 2008: Mitochondrial DNA phylogeography of the three-spined stickleback (Gasterosteus aculeatus) in Europe - Evidence for multiple glacial refugia. Mol. Phylogenet. Evol. 46: 167-182.

Merilä J., Crnokrak P. 2001: Comparison of genetic differentiation at marker loci and quantitative traits. J. Evol. Biol. 14: 892-903.

Molenberghs G., Verbeke G. 2005: Models for Discrete Longitudinal Data. Springer Series in Statistics. Springer-Verlag, New York, $687 \mathrm{pp}$.

Nielsen C.V., Buchmann K. 2001: Occurrence of Gyrodactylus parasites in Danish fish farms. Bull. Eur. Assoc. Fish Pathol. 21: $19-25$.

Raeymaekers J.A.M., Huyse T., Maelfait H., Hellemans B., VOLCKAERT F.A.M. 2008a: Community structure, population structure and topographical specialisation of Gyrodactylus (Monogenea) ectoparasites living on sympatric stickleback species. Folia Parasitol. 55: 187-196.

Raeymaekers J.A.M., Maes G.E., Audenaert E., Volckaert F.A.M. 2005: Detecting Holocene divergence in the anadromous-freshwater three-spined stickleback (Gasterosteus aculeatus) system. Mol. Ecol. 14: 1001-1014.

Raeymaekers J.A.M., Maes G.E., Geldof S., Hontis I., NackaErTs K., Volckaert F.A.M. 2008b: Modeling genetic connectivity in sticklebacks as a guideline for river restoration. Evol. Appl. 1: 475-488.

Raeymaekers J.A.M., Van Houdt J.K.J., Larmuseau M.H.D., Geldof S., Volckaert F.A.M. 2007: Divergent selection as revealed by $P_{\mathrm{ST}}$ and QTL-based $F_{\mathrm{ST}}$ in three-spined stickleback (Gasterosteus aculeatus) populations along a coastal-inland gradient. Mol. Ecol. 16: 891-905.

Reusch T.B.H., Wegner K.M., Kalbe M. 2001: Rapid genetic divergence in postglacial populations of threespine stickleback (Gasterosteus aculeatus): the role of habitat type, drainage and geographical proximity. Mol. Ecol. 10: 2435-2445.

Scharsack J.P., Kalbe M., Harrod C., Rauch G. 2007: Habitat-specific adaptation of immune responses of stickleback (Gasterosteus aculeatus) lake and river ecotypes. Proc. R. Soc. Lond. B, Biol. Sci. 274: 1523-1532. 
Soleng A., Bakke T.A. 1998: The susceptibility of three-spined stickleback (Gasterosteus aculeatus), nine-spined stickleback (Pungitius pungitius) and flounder (Platichthys flesus) to experimental infections with the monogenean Gyrodactylus salaris. Folia Parasitol. 45: 270-274.

Spitze K. 1993: Population structure in Daphnia obtusa: quantitative genetic and allozymic variation. Genetics 135: 367-374.

Van Dongen S., Lens L., Pape E., Volckaert F.A.M., RaeyMAEKERS J.A.M. 2009: Evolutionary history shapes the association between developmental instability and population-level genetic variation in three-spined sticklebacks. J. Evol. Biol. 22: 1695-1707.

Van Oosterhout C., Harris P.D., Cable J. 2003: Marked variation in parasite resistance between two wild populations of the Trinidadian guppy, Poecilia reticulata (Pisces: Poeciliidae). Biol. J. Linn. Soc. 79: 645-651.
Webster J.P., Gower C.M., Blair L. 2004: Do hosts and parasites coevolve? Empirical support from the Schistosoma system. Am. Nat. 164: S33-S51.

Wegner K.M., Kalbe M., Milinski M., Reusch T. 2008: Mortality selection during the 2003 European heat wave in threespined sticklebacks: effects of parasites and MHC genotype. BMC Evol. Biol. 8: 124.

Weir B.S., Cockerham C.C. 1984: Estimating F-statistics for the analysis of population structure. Evolution 38: 1358-1370.

Woolhouse M.E.J., Webster J.P., Domingo E., Charlesworth B., LEvin B.R. 2002: Biological and biomedical implications of the co-evolution of pathogens and their hosts. Nat. Genet. 32: 569-577.

Zietara M.S., Huyse T., Lumme J., Volckaert F.A.M. 2002: Deep divergence among subgenera of Gyrodactylus inferred from rDNA ITS region. Parasitology 124: 39-52.

Accepted 7 October 2010 